\title{
PLANETARY NEBULA EVOLUTION TRACED BY DISTANCE-INDEPENDENT PARAMETERS
}

\author{
C.Y. ZHANG \\ Department of Astronomy, The University of Texas at Austin, Austin, TX 78712 \\ and \\ S. KWOK \\ Department of Physics and Astronomy, The University of Calgary, Canada
}

\begin{abstract}
Making use of the results from recent infrared and radio surveys of planetary nebulae, we have selected 431 nebulae to form a sample where a number of distance-independent parameters (e.g., $\mathrm{T}_{b}, \mathrm{~T}_{d}, \mathrm{I}_{60 \mu m}$, and IRE) can be constructed. In addition, we also made use of other distance-independent parameters $\mathrm{n}_{e}$ and $\mathrm{T}_{*}$ where recent measurements are available. We have investigated the relationships among these parameters in the context of a coupled evolution model of the nebula and the central star. We find that most of the observed data in fact lie within the area covered by the model tracks, therefore lending strong support to the correctness of the model. Most interestingly, we find that the evolutionary tracks for nebulae with central stars of different core masses can be separated in a $\mathrm{T}_{b}-\mathrm{T}_{*}$ plane. This implies that the core masses and ages of the central stars can be determined completely independent of distance assumptions. The core masses and ages have been obtained for 302 central stars with previously determined central-star temperatures. We find that the mass distribution of the central stars strongly peaks at $0.6 \mathrm{M}_{\odot}$, with $66 \%$ of the sample having masses $<0.64 \mathrm{M}_{\odot}$. The luminosities of the central stars are then derived from their positions in the HR diagram according to their core masses and central star temperatures. If this method of mass (and luminosity) determination turns out to be accurate, we can bypass the extremely unreliable estimates for distances, and will be able to derive other physical properties of planetary nebulae.

This work was supported by a grant to SK from the Natural Sciences and Engineering Research Council of Canada. CYZ also acknowledges support by the NASA grant NAG 2-67.
\end{abstract}

